\title{
Nivel de conocimientos sobre determinantes de salud en estudiantes de grado de titulaciones relacionadas a la salud de la UGR
}

\section{Level of knowledge on health determinants in UGR students related to health}

\author{
Yuridia Díaz Casanova ${ }^{1}$ \\ ydiaz@uteq.edu.ec \\ Henry Aguilera Vidal ${ }^{1}$ \\ haguilera@uteq.edu.ec \\ Irene Bustillos Molina ${ }^{1}$ \\ ibustillos@uteq.edu.ec \\ Ruth Torres Torres ${ }^{1}$ \\ ritorres@uteq.edu.ec
}

Recibido: 1/07/2018, Aceptado: 1/09/2018

\begin{abstract}
RESUMEN
El estudio de los determinantes de la salud constituye un aspecto notable de la enseñanza que recibe el alumnado de titulaciones biosanitarias. El control de los problemas de salud pública requiere tomar conciencia de que existe un problema y sus causas, estar capacitados, conocer el sentido de los valores subyacentes en la comunidad y desear políticamente resolver el problema. Siendo así, se realizó un estudio observacional para apreciar el grado de conocimiento de estudiantes universitarios. Se planteó como objetivo estimar el grado de conocimiento que tienen los estudiantes universitarios de diferentes titulaciones biosanitarias sobre los determinantes de salud. Se aplicó un estudio observacional, transversal en una muestra de futuros profesionales sanitarios en las Facultades de Medicina, Ciencias de la Salud y Trabajo Social de la Universidad de Granada durante el curso académico 2015-2016. Se aplicó un cuestionario autoadministrado sobre determinantes de la salud. Los resultados mostraron que el estilo de vida es el factor que más influye sobre la salud seguido por la genética. En relación a los estilos de vida, la alimentación-actividad física es el principal influyente sobre la salud seguida por el consumo de drogas ilegales y el tabaco. En el grado de conocimiento sobre determinantes de salud se observa un déficit de conocimiento del alumnado sobre los factores sociales relacionados a la salud. Se sobreestima la importancia que la alimentación-actividad física y el tabaquismo tienen sobre la salud. Además, se sobrevalora la eficacia de la educación sanitaria en la lucha antitabáquica.
\end{abstract}

1 Docentes de la Universidad Técnica Estatal de Quevedo. Ecuador 


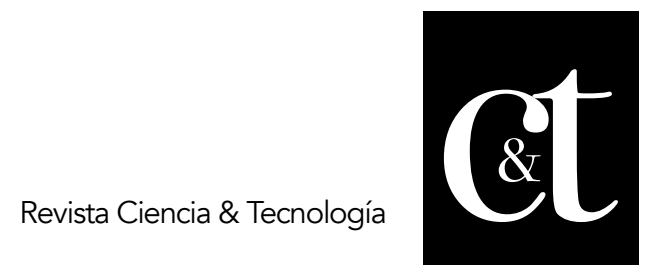

No. 20, 31 de octubre de 2018

ISSN impreso: 1390 - 6321

Palabras clave: salud pública, determinantes de salud, determinantes sociales de salud, estudiantes universitarios, titulaciones biosanitarias

\begin{abstract}
The study of health determinants is crucial in the learning process in undergraduate bio-sanitary programs. The control of public health problems demands the raising of the problem awareness, the understanding of its causes and being politically willing to face and solve it, based on a clear perception of the values assumed by the community. Bearing this in mind, an observational study has been carried out, in order to assess the university students' awareness of this issue. The objective set by this study was to estimate the level of knowledge of the students attending the different biomedical programs about health determinants. An observational crossstudy was applied to a sample of future healthcare professionals in the faculties of Medicine, Health Sciences, and Social Work, at Granada University during the 20152016 academic year. A self-administered questionnaire on health determinants, prepared by the authors and answered anonymously by the students was applied. Results, concerning the level of knowledge about health determinants, life style were observed to be the factors influencing health, followed by genetics, while the sanitary system followed by "other factors" showed the least influencing power. Concerning lifestyles, diet-physical activity is the main influencing factor, followed by consumption of illegal drugs and tobacco. In relation to the degree of knowledge on health determinants, the students showed a limited awareness of the social factors related to health. The importance of diet-physical activity and smoking for health is usually overestimated. The efficacy of sanitary education against smoking is overestimated as well.
\end{abstract}

Keywords: public health, health determinants, social health determinants, university students, biosanitary degrees

\title{
Introducción
}

El estudio de los determinantes de la salud establece un aspecto relevante de la enseñanza que recibe el alumnado de titulaciones biosanitarias, tales como medicina, enfermería, etc. El control de los problemas de salud pública requiere tomar conciencia de que existe un problema, entender sus causas, estar capacitados para hacer frente al mismo, conocer el sentido de los valores subyacentes en la comunidad y desear políticamente resolver el problema. Es labor de los profesores universitarios el conseguir que los alumnos tomen conciencia de los problemas de salud existentes y comprendan sus causas. Igualmente, deben transmitirles los conocimientos y herramientas básicas para hacer frente a los mismos. Únicamente cuando los estudiantes conozcan por qué se producen los problemas de salud, estarán capacitados para actuar sobre los mismos desde un punto de vista preventivo o curativo.

Especial relevancia tiene entre los determinantes de la salud los de naturaleza social, pues vienen a influir sobre el resto de determinantes modulándolos. Se entiende por determinante de la salud aquellas causas, condiciones o circunstancias que explican el nivel de salud de los individuos y poblaciones. Según la OMS, los factores determinantes de la salud se definen como el "conjunto de factores personales, sociales, políticos y ambientales que determinan el estado de salud de los individuos 


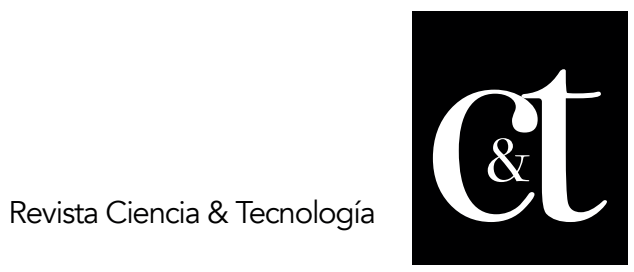

No. 20, 31 de octubre de 2018

ISSN impreso: 1390 - 6321

\section{y las poblaciones".}

Con frecuencia los determinantes se clasifican en heredados, es decir genéticamente determinados, y adquiridos, pero sus fronteras no están claramente delimitadas. Existe mucho por conocer en relación con los determinantes de salud, sobre todo aquellos que tienen que ver con la conducta de los individuos. Igualmente, es esencial comprender las interacciones entre los diferentes determinantes si queremos intervenir inteligentemente sobre los problemas de salud.

Según definiciones de la Organización Mundial de la Salud (OMS), Salud se refiere como un "estado de completo bienestar físico, mental y social, y no sólo la ausencia de afecciones o enfermedades" (Sánchez-González, 1998; OMS, 1948). Mientras que los factores determinantes de la salud se definen como el "conjunto de factores personales, sociales, políticos y ambientales que determinan el estado de la salud de los individuos y las poblaciones" (World Health Organization, 1998). En cuanto a los determinantes sociales "son aquellos factores y mecanismos por los cuales las condiciones sociales afectan el nivel de salud y sobre los que es posible intervenir a través de políticas sociales y de salud" (OMS, 1948).

Con el tiempo han surgido diferentes modelos de determinantes de salud, entre los que destacan; Modelo de Laframboise o Lalonde (Lalonde, 1974), Modelo de Tarlov (Tarlov, 1992). Por otro lado, los modelos de determinantes sociales de la salud surgen en los años 70 a partir de una serie de publicaciones que destacan las limitaciones de las intervenciones de salud orientadas a disminuir los riesgos individuales de enfermar y morir. De estos modelos, lo más destacados por mencionar son: Modelo de Diderichsen (Diderichsen, Evans, \& Whitehead, 2001), Modelo de Brunner, Marmot y Wilkinson (Acheson, 1998), Modelo de Solar e Irwin que caracterizan el contexto socioeconómico y político (Solar, Irwin, \& Vega, 2007), Modelo de Dahlgren y Whitehead que explica las inequidades en salud (Dahlgren \& Whitehead, 1991).

Se ha observado, que es frecuente que el alumno desconoce cuál es el principal factor de riesgo de enfermedad o infraestima la importancia de unos determinantes de salud en favor de otros. Este desconocimiento sobre los terminantes de salud suele ser mayor cuando hablamos de determinantes sociales, que con frecuencia ni siquiera son considerados por el alumno cuando es preguntado sobre los factores de riesgo de una determinada enfermedad. Por tal motivo, y dado que en la literatura revisada no hemos encontrado estudios que valoren, en estudiantes de centros universitarios que forman futuros profesionales sanitarios, el nivel de aprehensión de conocimientos sobre los determinantes relacionados con la salud. En el presente trabajo se hace una exploración para estimar el grado de conocimientos que tienen los estudiantes universitarios de diferentes titulaciones biosanitarias sobre determinantes de salud.

\section{Método}

Estudio observacional, transversal de corte realizado en una muestra de futuros profesionales sanitarios. Se estudió a 356 sujetos de las Facultades de Medicina, Ciencias de la Salud y Trabajo Social de la Universidad de Granada. El criterio de inclusión fue estar matriculado en una titulación que capacite para trabajar en sistema sanitario y tener aprobada o estar cursando la materia de salud pública 
बत

No. 20, 31 de octubre de 2018

ISSN impreso: 1390 - 6321

impartida por el Departamento de Medicina Preventiva y Salud Pública durante el curso académico 2015-2016. La población diana la compone el alumnado de los grados de Medicina, Enfermería, Fisioterapia, Terapia Ocupacional y Trabajo Social matriculado en asignaturas impartidas por el Departamento de Medicina Preventiva y Salud Pública. Concretamente se valoró a los alumnos de 5to. Curso de Medicina, 1ro. De enfermería, 3ro. De fisioterapia, 3ro de terapia ocupacional y 3ro de trabajo social. Para la recogida de información, todos los alumnos cumplimentaron de manera anónima un cuestionario de elaboración propia autoadministrado sobre determinantes de salud. El periodo de recogida de datos tuvo lugar entre el 16 de mayo al 13 de junio de 2016 y se garantizó que el alumnado había recibido información sobre determinantes de salud. Se utilizó el paquete estadístico Stata versión 14.0 para el análisis de la información.

\section{Resultados}

En cuanto al conocimiento sobre determinantes de la salud. La población finalmente estudiada está compuesta por un total de 356 sujetos de los que 47 son estudiantes de enfermería, 82 de medicina, 52 de fisioterapia, 29 de terapia ocupacional y 146 de trabajo social, lo que representa una tasa de participación del 21,9\%, 28,8\%, $88,1 \%, 39,7 \%$ y $66,4 \%$ respectivamente. Las titulaciones impartidas en la facultad de ciencias de la salud suman 128 participantes, tasa de respuesta del 36,9\%.

Por sexo, los varones representan un $30,1 \%$ frente al $69,9 \%$ de mujeres, siendo la edad media de 23 años (DE 4.46 años). Por tamaño del lugar de residencia, la muestra se repartió por igual entre las tres categorías consideradas.

Aproximadamente, un tercio de los estudiantes refirió que sus progenitores solo habían realizado estudios primarios, el $29,34 \%$ en el caso del padre y el $28,17 \%$ en el caso de la madre. Encuestados por la profesión de sus progenitores, el 11,36\% refirió que su padre tenía una profesión relacionada con la salud, porcentaje asciende a casi el doble $(19,49 \%)$ cuando se les preguntó por las madres. Respecto a la formación recibida en estudios de grado, médicos (media $=3,69$ ) y estudiantes de ciencias de la salud (media $=4,17$ ) fueron quienes dieron puntuaciones más bajas a la enseñanza recibida relacionada con factores sociales, mientras que esa última posición fue ocupada por los factores genéticos en el caso de los trabajadores sociales (media $=3,05)$.

De los 20 factores analizados, el alcohol (media $=4,7$ ) y el tabaco (media $=4,7$ ), seguidos por la alimentación, la actividad física y las drogas ilegales fueron aquellos que los alumnos refirieron como los más abordados durante sus estudios; los factores que menos interés recibieron durante sus estudios fueron la religión (media $=2,4$ ), los valores éticos (media $=3,2$ ), la soledad (media $=3,4$ ) y la vivienda (media $=3,4$ ). Por titulación, es el tabaco el factor sobre el que más información dicen recibir los alumnos de medicina y ciencias de la salud mientras que los trabajadores sociales consideran que se presta más atención a las relaciones sociales y familiares en su enseñanza.

Grado de conocimiento sobre determinantes de la salud: Global (ver Tabla 3)

Al ser preguntados los estudiantes sobre el factor que más influye sobre la salud $(p<0.05)$, el $83,2 \%$ puso en primer lugar a los estilos de vida, seguidos por la 


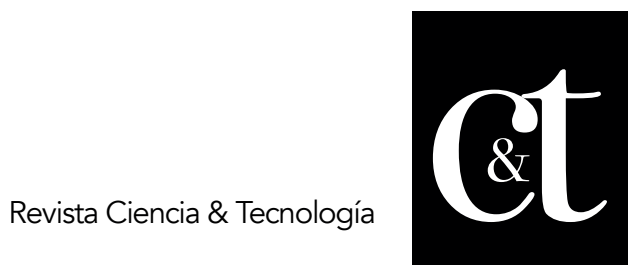

No. 20, 31 de octubre de 2018

ISSN impreso: 1390 - 6321

genética con un $9,6 \%$. Cuando la pregunta se refirió al factor que menos influía $(\mathrm{p}<0.16)$, la primera posición fue ocupada por el sistema sanitario $(37,3 \%)$ seguida por "otros factores" $(36,2 \%)$.

En relación a los estilos de vida, la alimentación-actividad física $(p<0.12)$ ocupó la primera posición como factor influyente sobre la salud $(43,9 \%)$, seguida por el consumo de drogas ilegales $(p<0.05)(29,6 \%)$ y el tabaco $(p<0.05)(22,25 \%)$. La alimentación-actividad física $(p<0.05)$ y el consumo de tabaco $(p<0.05)$ fueron los dos factores citados en mayor número de ocasiones por el alumnado entre las tres primeras posiciones, ambos con un porcentaje del $76,1 \%$, siendo el menos citado los hábitos de conducción $(p<0.05)$ con un $9,8 \%$.

En consonancia con los resultados anteriores, la promoción de la actividad física (media $=5,2$ ) y de una alimentación sana (media $=5,4$ ) fueron las actuaciones consideradas por los estudiantes como más influyentes para mejorar la salud de la población, siendo la reducción de la tasa de paro (media $=4,2$ ) y de las desigualdades sociales (media $=4,8$ ) los factores que menos importancia les merecieron.

Cuando se les preguntó específicamente por las medidas que consideraban más eficaces para combatir el tabaquismo, fue la educación sanitaria la que ocupó la primera posición (media $=4,8$ ) seguida por el incremento del precio (media $=4,5$ ), mientras que la prohibición de la publicidad se situó como la actividad más ineficaz (media $=3,6$ ).

Por último, se trató de indagar en este bloque el conocimiento de los estudiantes sobre las principales causas de mortalidad en nuestro medio resultando ser para el $51,1 \%$ de los alumnos las enfermedades cardiovasculares la causa más importante, seguidas por el cáncer con un 22,5\%. En cambio, fue el cáncer la causa más citada entre las tres primeras posiciones, por delante de las enfermedades cardiovasculares.

Grado de conocimiento sobre determinantes de la salud: Por titulación cursada (ver Tabla 4)

A la pregunta de cuál es el factor que más influye sobre la salud, los futuros médicos contestaron que son los estilos de vida $(98,8 \%)$, porcentaje que contrasta con el $69,2 \%$ obtenido para los trabajadores sociales. También llama la atención que ningún estudiante de medicina dijera ser la genética o el sistema sanitario el factor más influyente. Por su parte, para todos los estudiantes, independientemente de su titulación, el factor que menos importancia tiene es el sistema sanitario y con porcentajes relativamente similares en torno al $40 \%$, diferencias que no fueron estadísticamente significativas.

Cuando se cuestionó por el principal estilo de vida que influye sobre la salud, hubo consenso al situar en primer lugar a la alimentación-actividad física. En cambio se mostraron diferencias en cuanto al segundo factor citado, que en el caso de los estudiantes de medicina fue el tabaco y para las otras dos titulaciones las drogas ilegales, relegando al tabaco a la tercera posición. Este hecho se corrige en el caso de los estudiantes de ciencias de la salud cuando se valora la variable "haber situado el factor entre las tres primeras posiciones" pero no ocurre lo mismo con los trabajadores sociales para los que incluso las drogas ilegales pasan a ocupar la 


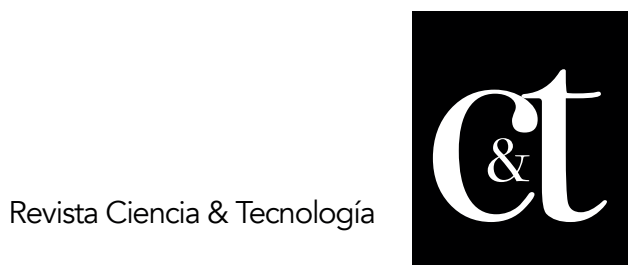

No. 20, 31 de octubre de 2018

ISSN impreso: 1390 - 6321

primera posición $(80,8 \%$ para consumo de drogas frente a $72,6 \%$ para la alimentación-actividad física); en cambio, para médicos y estudiantes de ciencias de la salud el tabaco pasa a ocupar la primera posición.

Cuando se preguntó por la actuación que consideraban más importante para mejorar la salud, todos coincidieron en ser la promoción de la alimentación saludable y de actividad física, con valores medios en torno al 5,5. Únicamente cabe destacar la importancia que dan los estudiantes de trabajo social al incremento del gasto sanitario como manera de mejorar la salud poblacional (media =5,14), actuación que fue considerada la menos importante por los futuros médicos (media=4).

Respecto a cuál medida consideraban más eficaces para combatir el tabaquismo, todos coincidieron en situar a la educación sanitaria en primera posición y a la prohibición de la publicidad en última posición pero con pesos distintos en función de la titulación. Para la educación sanitaria la mayor puntuación se obtuvo con los estudiantes de medicina y la menor con los trabajadores sociales. Para ambas variables las mayores puntuaciones fueron para los estudiantes de medicina y las menores para los estudiantes de trabajo social.

\section{Discusión}

En general, el grado de conocimiento sobre los grandes bloques de determinantes de la salud entre los estudiantes es adecuado. Así, todos ellos, independientemente de la titulación, señalan a los estilos de vida como los más importantes y al sistema sanitario como el menos importante en consonancia con lo descrito por Dever en su conocido trabajo "An epidemiological Model for Healh Policy Analysis" de 1976. Es destacable, pero esperable, que sean los futuros trabajadores sociales los que con menos frecuencia señalan como principal determinante a los estilos de vida, probablemente resultado de su plan de estudios, muy diferente al del resto de titulaciones analizadas.

Es la misma circunstancia antes mencionada la que explica que mientras los estudiantes de medicina y ciencias de la salud tienen claro que los estilos de vida que más influyen son la alimentación-actividad física y el tabaquismo, los de trabajo social consideran en primer lugar al consumo de drogas ilegales. Destaca que cerca del $15 \%$ de los alumnos de medicina y el $25 \%$ de los de enfermería no señalen la alimentación-actividad física como uno de los tres primeros factores en importancia, porcentajes que son del 8 y del $20 \%$ para el caso del tabaco respectivamente. Esta circunstancia es especialmente relevante e indicativa de que algo falla en la enseñanza que reciben estos alumnos.

Tampoco tienen claro los estudiantes el que son el aumento de precio y la prohibición de la publicidad las medidas que se han demostrado más eficaces para combatir el tabaquismo. Así todos los alumnos, independientemente de la titulación, señalaron como medida más eficaz la educación sanitaria que, a pesar de su importancia, se ha comprobado que tiene eficacia limitada dada la edad y circunstancias existentes cuando se empieza a fumar. En cambio señalaron como la menos eficaz la prohibición de la publicidad. El entender lo "prohibido" como algo malo y que no debe hacerse constituye una manera de pensar entre la juventud que no es siempre acertada. 


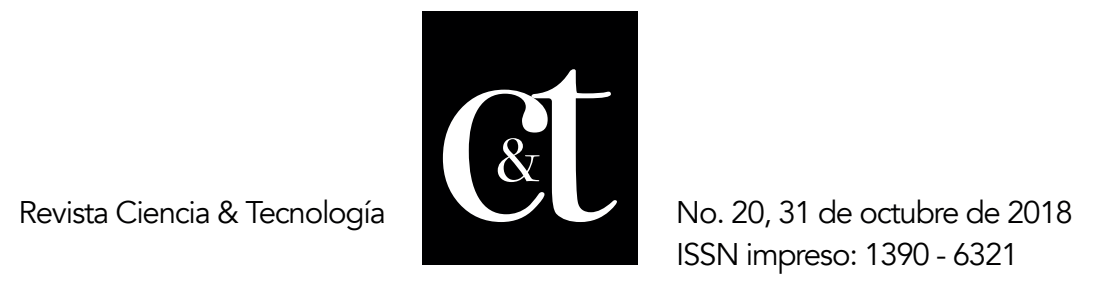

\section{Referencias bibliográficas}

Acheson, D. (1998). Independent inquiry into inequalities of health. London: The Stationery Office.

Dahlgren G. \& Whitehead M. (1991). Policies and strategies to promote social equity in health. Stockholm, Sweden: Institute for Futures Studies.

Dever G. (1976). An epidemiological model for health policy analysis. Soc Ind Res 1976; 2: 453-66.

Diderichsen, F., Evans, T., \& Whitehead, M. (2001). The social basis of disparities in health. Challenging inequities in health: From ethics to action, Vol. 1: 12-23.

Lalonde, M. (1974). A new perspective on the health of Canadians. Minister of Supply and Services Canada. Retrieved from Public Health Agency.

OMS (1948). Constitución de la Organización Mundial de la Salud.

Sánchez-González, M.A. (1998). El concepto de salud: Análisis de sus contextos, sus presupuestos y sus ideales. En Cuadernos del Programa Regional de Bioética, No 7, Programa Regional de Bioética para América Latina y el Caribe OPS/OMS, Santiago de Chile.

Solar O., Irwin A., \& Vega J. (2005). Equity in Health Sector Reform and Reproductive Health: Measurement Issues and the Health Systems Context. WHO Health Equity Team working paper.

Tarlov, A. R. (1992). The coming influence of a social sciences perspective on medical education. Academic Medicine, November 1992 - p 724-31.

World Health Organization - WHO (1998). The WHO Health Promotion Glossary. Geneva. 


\section{ANEXOS}

\begin{tabular}{|c|c|c|c|c|c|c|c|c|c|}
\hline \multirow[t]{2}{*}{ Variable } & & \multicolumn{2}{|c|}{ Global } & \multicolumn{2}{|c|}{ Medieina } & \multicolumn{2}{|c|}{ coss } & \multicolumn{2}{|c|}{15} \\
\hline & & $\mathrm{N}$ & $\%$ & N & 96 & N & $\%$ & M & $\%$ \\
\hline \multicolumn{10}{|c|}{ Sexo $(p=0,695)$} \\
\hline & Varón & 107 & 30.06 & 24 & 2927 & 42 & 3281 & 41 & 2805 \\
\hline & Mesjer & 249 & 59.94 & 5\$. & 70.75 & 86 & 6719 & 105 & 71.92 \\
\hline \multicolumn{10}{|c|}{ Edad $(p<0,05)$} \\
\hline & $\leq 21$ añes & 150 & 4213 & 1 & $1: 22$ & 86 & 67.19 & 63 & 4315 \\
\hline & $23-25$ años & 162 & 45.51 & 76 & 92.68 & $2 ?$ & 21.09 & 59 & 40.41 \\
\hline & $>25$ andos & 44 & 12.36 & 5 & 6.10 & is & 1172 & 24 & 16.44 \\
\hline \multicolumn{10}{|c|}{$N^{*}$ bab. Resideneia $\left(p^{-0} 0,05\right)$} \\
\hline & $<20,000$ & 132 & 37,08 & 13 & 1585 & 62 & 48.44 & 57 & 39.04 \\
\hline & $20,000-100.000$ & 123 & 34.55 & 21 & 25.61 & it & 36.72 & 55 & 37.67 \\
\hline & $5-100,000$ & 101 & 2837 & 48 & 58,54 & 16 & 1484 & 34. & 23,29 \\
\hline \multicolumn{10}{|c|}{ Esfudios padre $(\mathrm{p}<0.05)$} \\
\hline & Primasos & 104 & 29,34 & 9 & 1111 & 38 & 29.69 & 57 & 39.04 \\
\hline & Secubdarios: & 120. & 36.34 & 16 & 19.75 & 55 & 4297 & 58 & 3973 \\
\hline & Dhiversiturios & 122 & 34.37 & 56 & 69.14 & 35 & $27: 34$ & II & 21.23 \\
\hline \multicolumn{10}{|c|}{ Estadios madre $\left(p^{-0,05}\right)$} \\
\hline & Prinarias & 100 & 28.17 & 11 & 13,58 & 13 & 3359 & 46 & $31: 51$ \\
\hline & Secundarios & 135 & 38.03 & 17 & 20.99 & 51 & 39.84 & 67 & 45.89 \\
\hline & Uasveritarios & 120 & 33.80 & 53 & 65.43 & 34 & 26.56 & 33 & $22 m$ \\
\hline \multicolumn{10}{|c|}{ Profosiòe padre $(p<0,05)$} \\
\hline & No & it: & 84.64 & 63 & 77,78 & 111 & 88,80 & 138 & 9452 \\
\hline & Si & 40 & 11.56 & 18 & 22.22 & 14 & 11.20 & is & 5,48 \\
\hline \multicolumn{10}{|c|}{ Profesión madre (p 0,05$)$} \\
\hline & No & 235 & 80,51 & 57 & 70.37 & 106 & 32.51 & 122 & 8414 \\
\hline & Si & 69 & 10.49 & 24 & 29,63 & 22 & 1719 & 25. & 15.86 \\
\hline \multicolumn{10}{|c|}{ Fauniliar TS $(\mathrm{p}-0,05)$} \\
\hline & No & 249 & 69.94 & 60 & 73.27 & 98. & 76.56 & 91 & 6235 \\
\hline & 5i & 107 & 30.06 & 22 & 26,83 & 30 & 23.44 & 35 & 37.67 \\
\hline \multicolumn{10}{|c|}{ Conociniento Is fpce,05) } \\
\hline & No & 132- & 37.08 & 32 & 39.02 & 73 & 57,03 & 27 & $18-49$ \\
\hline & Si & 224. & 62.92 & 50 & 60.98 & 55. & 42.97 & 119 & 8151 \\
\hline
\end{tabular}

$\mathrm{N}=$ Frecuencia absoluta: TS=Trabajador social: Eustre paréatesis fienara valor $p$ (Chi)

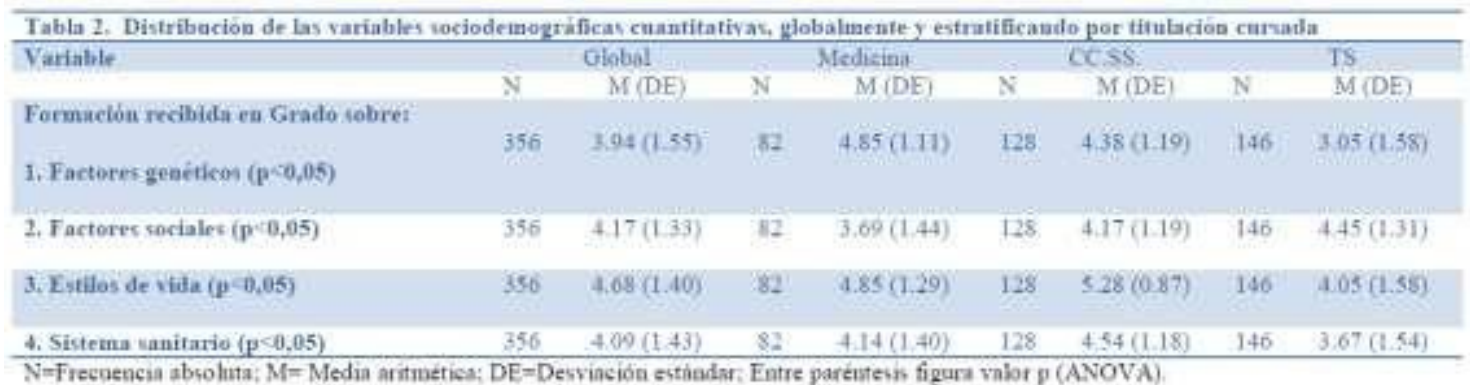




\begin{tabular}{|c|c|c|c|c|c|c|c|c|}
\hline \multirow[b]{2}{*}{ Variable } & \multicolumn{2}{|r|}{ Global } & \multicolumn{2}{|c|}{ Medicina } & \multicolumn{2}{|c|}{ cess: } & \multicolumn{2}{|r|}{ TS } \\
\hline & $\mathrm{N}$ & $M(D E)$ & N & $\mathrm{N}(\mathrm{DE})$ & $\mathrm{N}$ & $M(D E)$ & $\mathrm{x}$ & $\mathrm{M}(\mathrm{DE})$ \\
\hline \multicolumn{9}{|l|}{ Formación recibida en Grado sobre: } \\
\hline 1. Relaciases famillares $(p-0,05)$ & 356 & $3.75(1.43)$ & 82 & $3.19(1.29)$ & 128 & $3 ; 54(1 ; 33)$ & 146 & $423(1.44)$ \\
\hline 2. Rrlariones soeiales $(\mathrm{\beta}<0,05)$ & 356 & $4.01(0.33)$ & 82 & $3.40(1.33)$ & 128 & $3.86(1.25$ & 146 & $4.47(1.24)$ \\
\hline 3. Soledad $(\mathrm{p} c 0,05)$ & 356 & $335(1.57)$ & 82 & $2.65(1.63)$ & 128 & $3.24(1.54)$ & 146 & $3.84(1.40)$ \\
\hline 4. Alimeatariòn ( $p c 0,05$ ) & 356 & $4.55(1.64)$ & 82 & $5.07(1.13)$ & 129 & $5.36(0.92)$ & 146 & $3.56(1.84)$ \\
\hline 5. Cupacidad Adqquisitiva & 356 & $3.40,41)$ & 182 & $3.51(1.38)$ & 125 & $5.35(1.41)$ & 146 & $3,47(1.45)$ \\
\hline 6. Caracteristiras de la vivienda (p 0,05$)$ & 356 & $3.39 .(1.53)$ & 82 & $2.80(1.64)$ & 128 & $3.12(1.40)$ & 146 & $3.96(137)$ \\
\hline 7. Sivel educarive $(\beta=0,70)$ & 356 & $4.01(1.37)$ & 82 & $4.23(1.26)$ & 128 & $5.96(1.39)$. & 146 & $3.94(1.41)$ \\
\hline 8. Tahaquicumo (p'0,05) & 356 & $4.68(1.79)$ & 82 & $5.78(0.49)$ & 128 & $5.59(0.86)$ & 146 & $3.27(1.92)$ \\
\hline 9. Actividad física $(p<0,05)$. & 356 & $4.62(1.70)$ & 82 & $5.48 \times 0.831$ & 128 & $5.59(0.84)]$ & 146 & $3.34(1.82)$ \\
\hline 10. Prufesión $\left(p^{-10}, 15\right)$ & 356 & $3.54(1.36)$ & 82 & $356(1.23)$ & 128 & $3,67(132)$ & 146 & $3.43(1.47)$ \\
\hline 11. Clase social (p<0,05) & 356 & 3.8761 .405 & 82 & $3.75(1,21)$ & 138 & $3: 54(1.41)$ & 146 & $421(1.41]$ \\
\hline 12. Lugar de resideneia ( $p<0.05$ ) & 356 & $3.56(1.47)$ & 82 & $3.25(1.36)$ & 128 & $3.42(1.51)$ & 146 & $3.85(1.44)$ \\
\hline 13. Valeres étices $(p<0.05)$ & 356 & $323(173)$ & 82 & $214(1.65)$ & 123 & $3.48(1.60)$ & 1.46 & $3,61 \div 1.66)$ \\
\hline 14. Cultura ( $\mathrm{p}<0,015$ ) & 356 & $3,00(1,54)$ & 82 & $2.73(1.48)$ & 128 & $3.72(1.42)$ & 146 & $399(1.49)$ \\
\hline 15. Drogas itegales $(p<0,05)$ & 356 & $4.42(1.53)$ & 82 & $4.5 .5(1.95)$ & 128 & $4.837(1.32)$ & 146 & $3.99(1.69)$ \\
\hline 16. Actividades de ocio $(\mathrm{p}<0,05)$ & 356 & $3.55(1.49)$ & 82 & $3.10(1.36)$ & 128 & $3.99(14 \theta)$ & 140 & $3.38(156)$ \\
\hline 17. Nleaholismo $(p<0.05)$ & 356 & $473(1,41)$ & 82 & $5,32(0.87)$ & 128 & $5.17(1.01)$ & 146 & $702(1.64)$ \\
\hline 18. Religien ( $\mathrm{p}-0,05)$ & 356 & $2.40(1.77)$ & 82 & $1,39(1.40)$ & 128 & 2.7101 .711 & 146 & $2.69(1.80)$ \\
\hline 19. Sueuิe $(\mathrm{p}<0.05)$ & 356 & $3,40(1.71)$ & 82 & $378(1,46)$ & 128 & $3.88(1.55)$ & 146 & $276(\mathrm{t}, 75)$ \\
\hline 20. Contamisaciòn atmesférica $(\mathrm{p} \subset 0,05)$ & 356 & $3.51(1.78)$ & 82 & $413(132)$ & 128 & $3.78(1.65)$ & 146 & $293(196)$ \\
\hline
\end{tabular}

\begin{tabular}{|c|c|c|c|c|c|c|c|c|c|}
\hline \multirow[b]{2}{*}{ Variable } & & \multicolumn{2}{|c|}{ Global } & \multicolumn{2}{|c|}{ Modicma } & \multicolumn{2}{|c|}{ COSS: } & \multicolumn{2}{|c|}{ IS } \\
\hline & & $\mathrm{N}$ & $\%$ & N & $\%$ & $\mathrm{~N}$ & is & N & \% \\
\hline \multicolumn{10}{|c|}{ QQud factor influye mas sobre ta salud? } \\
\hline & 1. Gençicn & 34 & $955:$ & 0 & 0.00 & 8 & 6.25 & 26 & 17.81 \\
\hline & 2. Medio subtiente & 15 & 421 & 1 & 1.22 & 3 & 234 & Ii & 7.83 \\
\hline & 3. Estilos de vids & 290 & 83.15 & 81 & 98,75 & 114 & 89.06 & 101 & 69.15 \\
\hline & 4. Sblentua vatuitario & ?. & 1,97 & 0 & 000 & E & 6.28 & 6 & 411 \\
\hline & 5. Otro & 4 & 1.12 & 0 & 0.00 & 2 & 1.56 & 2 & $1.3 ?$ \\
\hline \multicolumn{10}{|c|}{$\begin{array}{l}\text { ¿Quí factor influye meaos sobre la salud? } \\
(\rho-0,16)\end{array}$} \\
\hline & 1. Gentricn & 54 & 15.25 & 16 & 19,31 & 18 & 14.29 & 20 & 13,70 \\
\hline & 2. Medio smbiecte & 34 & $9.80^{\circ}$ & $7^{\circ}$ & 3.66 & 18 & 1419 & is & 890 \\
\hline & 1. Evtilos de vida & 6 & 1.69 & 1 & 1.22 & 1 & 0.79 & 4 & 2.74 \\
\hline & 4 Sthtemi subitario & 132 & $32 \geq 0$ & 35 & 41.68 & 48 & 38.10 & 49 & 33.56 \\
\hline & 5. Otro & 128 & 36,16 & 27 & 32,93 & 41 & 32,54 & 60 & 41.10 \\
\hline
\end{tabular}

$\mathrm{N}-\mathrm{Frtcuencia}$ absolata. Entre partatesis figura valor $p$ (Chi) 


\begin{tabular}{|c|c|c|c|c|c|c|c|c|c|}
\hline \multirow[b]{2}{*}{ Variable } & & \multicolumn{2}{|c|}{ Global } & \multicolumn{2}{|c|}{ Medicusb. } & \multicolumn{2}{|c|}{ CCSS. } & \multicolumn{2}{|c|}{ IS } \\
\hline & & N & $\%$ & $\mathrm{~N}$ & sio & $\mathrm{N}$ & $\%$ & N & 4 \\
\hline \multicolumn{10}{|c|}{ 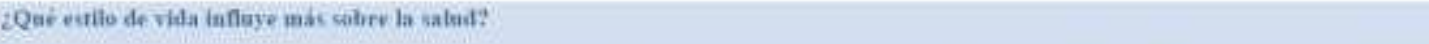 } \\
\hline & 1. Alimentaciòn y act. Eisica $(p-0,12)$ & 158 & 43,9: & 39 & $47 \$ 6$ & 50 & 3937 & 62 & 4589 \\
\hline & 2. Cousurus de tabaso $(\mathrm{p}<0.05)$ & 79 & 2225 : & 35 & 42.68 & 32 & 25,20 & 12 & 5.22 \\
\hline & 3. Consrmo de abolio! $(p=0,10)$ & 9 & 2.34 & I & 1.22 & 2. & 157 & 6 & 411 \\
\hline & 4 Consumo de dropis $(\mathrm{p}<0,05)$ & 105 & 29.58 & 7 & 8.54 & 39 & 30.71 & 59 & 70,41 \\
\hline & 5. Habitos sexuales (pa 0.10$)$ & 1 & 0.28 & (2) & 0.20 & () & 0.00 & 1 & 0.68 \\
\hline & 6. Hábitos de condueciôn (p<0,05) & 5 & 1.41 & 0 & $0-00$ & 4 & 3.15 & 1 & 0.68 \\
\hline \multicolumn{10}{|c|}{ Estile situado eatre los 3 primeros por of estudiante } \\
\hline & 1. Almimentación y act. fisica $p(0,05)$ & 271 & 76.12 & 71 & 86.59 & 94 & 73,44 & 106 & 72.60 \\
\hline & 2. Consumo de tibase $p<0.05)$ & 271 & 7612 & 77 & 93.90 & 104 & $81: 25$ & 90 & 61.04 \\
\hline & 3. Consunb de akobiol p $c 0,05$ ) & 216 & 60.67 & 59 & 71.05 & 66 & $\$ 1.56$ & 91 & 62,33 \\
\hline & + Conssume de drogas $p$ c0.05) & $221 \mathrm{H}$ & 64.04 & 27 & 32.93 & 83 & 64,84 & 118 & 80.82 \\
\hline & 5. Hatito vexuale $p-0,13$ ) & 42 & $11=0$ & 10 & 12.20 & i4 & 10.94 & 18: & 1233 \\
\hline & 6. Habitos de cooducción $\mathrm{p}<0,05\}$ & 35 & 9.83 & 2 & 2.44 & 18 & 14.06 & 15 & 10.27 \\
\hline
\end{tabular}

$\mathrm{N}=$ Frecuencia abrolita: Enire parentesis frgan valor $p$ (Chii)

\begin{tabular}{|c|c|c|c|c|c|c|c|c|}
\hline \multirow[b]{2}{*}{ Variable } & \multicolumn{2}{|r|}{ Global } & \multicolumn{2}{|r|}{ Medreina } & \multicolumn{2}{|r|}{ cess. } & \multirow{2}{*}{\multicolumn{2}{|c|}{ IS }} \\
\hline & $\mathrm{N}$ & $M(D E)$ & $\mathrm{N}$ & $M(D E)$ & $\mathrm{N}$ & $\mathrm{M}(\mathrm{DE})$ & & \\
\hline \multicolumn{9}{|l|}{$\begin{array}{l}\text { Que actuación consideras que influye más } \\
\text { sobre la salud de la poblacion? }\end{array}$} \\
\hline 1. Reducción de la tasa de paro $(\mathrm{y}=0.52)$ & 356 & $4.24(1.18)$ & 82 & $+37(1,01)$ & 128 & $4.21(1.14)$ & 146 & $4.20(1.33)$ \\
\hline 2. Mis gasto wu sirtema sasitario (p 0.05 ) & 356 & $4.75(120)$ & 82 & $4(1.27)$ & 128 & $488(1.10)$ & 146 & $5.14(1.03)$ \\
\hline 3. Redecir designtaldades sociales (p-0,05) & 356 & $4.760 .10)$ & 82 & $531(0,78)$ & 128. & $434(1.17)$ & 146 & $4.82(1.05)$ \\
\hline 4. Promecion astividad fisica ( $p<0,05$ ) & 356 & $5,16(0,97)$ & 82 & $5.46(0.74)$ & $12 h$ & $5.35(0.87)$ & 146 & $4.84(1.07)$ \\
\hline 5. Promeción alimentación sana (p:0,05) & 356 & $5.39(0.90)$ & 82 & $5.5 .4(0.73)$ & 128 & $5.5(0.87)$ & 146 & $5.22(0.98)$ \\
\hline
\end{tabular}

\begin{tabular}{|c|c|c|c|c|c|c|c|c|c|}
\hline \multirow[b]{2}{*}{ Variable } & ( & \multicolumn{2}{|c|}{ Clobai } & \multicolumn{2}{|c|}{ Medicina } & \multicolumn{2}{|c|}{ coss } & \multicolumn{2}{|c|}{ TS } \\
\hline & & $\mathrm{N}$ & $\%$ & $\mathrm{~N}$ & $\because$ & $\mathrm{s}$ & sis & $\mathrm{N}$ & 4 \\
\hline \multicolumn{10}{|c|}{2 Cuat es la principal caina de muerte en Espaita? } \\
\hline & 1. Violencia de eeners (pc0.05) & 20 & 5,62 & 32 & 39.02 & 70 & 54.69 & 28 & 10.18 \\
\hline & 2.5 sucvdis $\left(\mathrm{p}^{<0,05}\right)$ & 15 & +21 & 21 & 25.61 & 35 & 27.34 & 33 & 22.0 \\
\hline & 3. Aecidentes is tráfico (pc0,a5) & 51 & 1432 & 15 & 1829 & 9 & 7.07 & 22 & 15.07 \\
\hline & 4 Canser $(p<0,05)$ & 80 & 22.47 & 1 & 1.22 & 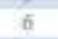 & 4.69 & 3 & 2.05 \\
\hline & 5. Enfi rsupiraterias (p<0,05) & 8 & 2,25 & 13 & 15.85 & 5 & 391 . & 35 & 23,97 \\
\hline & 6. Enf cardiovasculares ( $\mathrm{p}<0,(15)$ & 182 & 51.12 & 0 & 0.00 & 3 & 234 & 25 & 17.12 \\
\hline \multicolumn{10}{|c|}{ Causa situada eutre las 3 primeras por el estudiante } \\
\hline \multicolumn{10}{|c|}{ Oancer $(\mathrm{p}=0,34)$} \\
\hline & & 301 & 84.55 & 68 & 82.93 & 110. & 85.94 & 123 & $8+25$ \\
\hline & Enf respiratorias ( $(y=0,05$ ) & 171 & 48.03 & 37 & 45.12 & 78 & 60.94 & 56 & 38.36 \\
\hline & Enf cartiovisculares ( $\mathrm{p}<0,05)$ & 275 & 77.25 & 78 & 95.12 & 120 & 93.75 & 77 & 52.74 \\
\hline
\end{tabular}

